\title{
Application of the Riccati hereditary mathematical model to the study of the dynamics of radon accumulation in the storage chamber
}

\author{
Dmitryi Tverdyi ${ }^{1 *}$, Roman Parovik ${ }^{1,2}$, Evgeniy Makarov ${ }^{1,3}$, Pavel Firstov $^{3}$ and Nazira \\ Alimova $^{4}$ \\ ${ }^{1}$ Vitus Bering Kamchatka State University, 683032 Petropavlovsk-Kamchatskiy, Russia \\ 2 Institute of Cosmophysical Research and Radio Wave Propagation FEB RAS, 684034 Paratunka, \\ Kamchatka, Russia \\ ${ }^{3}$ Kamchatka Branch of the Federal State Budgetary Institution of Science of the Federal Research \\ Center "Unified Geophysical Service of the Russian Academy of Sciences", 683006 Petropavlovsk- \\ Kamchatskiy, Russia \\ ${ }^{4}$ National University of Uzbekistan named after Mirzo Ulugbek, 100174 Tashkent, Uzbekistan
}

\begin{abstract}
The article proposes a mathematical model of radon accumulation in a chamber, which takes into account the hereditary properties of the environment in which radon migrates. The model equation is the fractional Riccati equation with a derivative of a fractional variable order of the Gerasimov-Caputo type, taking into account heredity, as well as taking into account nonlinearity, which is responsible for the mechanisms of radon entry into the chamber. The obtained model curves of the accumulation process are compared with real data. It is shown that the model described in the work gives a better agreement between the model and real curves of radon accumulation and can be used for a more accurate description of the processes occurring in the chamber.
\end{abstract}

The article is dedicated to the memory of the author, our colleague P.P. Firstov.

\section{Introduction}

Observation of the radon field in the upper soil layer is of interest both in connection with the biological activity of this gas during accumulation in basements and on the first floors of buildings, and from the point of view of developing a method for predicting strong earthquakes based on continuous monitoring of the volumetric activity of radon in the subsoil air with a high degree of detail. The radon runoff into the atmosphere is influenced by changes in meteorological values, water cut and freezing of the upper soil layer, changes in the stressstrain state of the geomedium.

According to modern concepts, variations in regional stress fields associated with the preparation of the future earthquake source lead to a change in porosity, permeability,

* Corresponding author: dimsolid95@gmail.com 
temperature and pressure gradients in the upper layer of the earth's crust and, as a consequence, to a change in the rate of radon migration to the day's surface. Thus, monitoring of the parameters of the radon field at the boundary of the lithosphere - atmosphere allows us to talk about the processes occurring in the earth's crust, which can cause anomalous variations in the concentration of radon, as well as a change in the flux density of RFD (Radon Flux Density) from the soil surface [1,2].

Since 1997, at the Petropavlovsk-Kamchatsky geodynamic test site, monitoring of the volumetric activity of radon $(\mathrm{Rn})$ has been carried out in order to search for precursors of strong earthquakes [3].

In this paper, using mathematical modeling, the process of radon accumulation in a chamber with gas-discharge meters is investigated to determine RFD from the soil surface below it.

The concentration of radon inside chambers installed on the ground is the result of two main competing processes: radon influx and radon efflux. In this case, the processes are due to the presence of a radon concentration gradient in the medium and a pressure gradient. In the first case, a diffusion transfer process is formed, in the second - convective (gas transfer in a gas) or filtration (gas transfer in a porous medium).

When organizing radon monitoring points, it is necessary to know the RFD value from the surface at the selected point where the camera will be installed. In addition, areas of increased radon discharge into the atmosphere are characterized by narrowly localized zones, the search for which determines the need for a quick RFD assessment using a simple and affordable method. This method, based on the mathematical description of the accumulation of $\mathrm{Rn}$ in the chamber according to the approach from [4], is described in detail in [3] and was tested at the monitoring points of subsurface gases in Kamchatka [3].

In the mathematical description of the radon accumulation process in the chamber in [3], a number of assumptions were used to simplify the solution of the problem, and the accumulation process itself was assumed to be stationary when there are no abruptly changing AER (Air Exchange Rate) and RFD conditions from the surface below it. Consequently, possible factors affecting the flow rate were not taken into account, primarily changes in atmospheric pressure and temperature. In the chamber used in the work, air exchange with atmospheric air is present, but minimized. Due to weather conditions (mainly due to temperature fluctuations), the AER will vary significantly.

For a more accurate description of the accumulation process, the existing model was modified using a nonlinear function responsible for the mechanisms of Rn entry into the chamber, as well as taking into account heredity.

\section{Mathematical model}

Mathematical modeling of the transfer of $\mathrm{Rn}$ in porous soil due to the mechanisms of diffusion and convection, taking into account the hereditary properties of the medium, is considered in $[5,6]$. Such mechanisms of $\mathrm{Rn}$ transfer in the literature are called subdiffusion and superdiffusion or anomalous diffusion [7]. In this work, we will consider a slightly different hereditary process of radon migration in the chamber.

Let us consider the following law of accumulation of volumetric activity of radon RVA (Radon Volumetric Activity) in the chamber:

$$
\frac{d A}{d t}=\int_{0}^{t} K(t-\sigma) F(A, \sigma) d \sigma, \quad A(0)=A_{0},
$$

where $A(t)$ - RVA, Bq $/ \mathrm{m}^{3} ; F(A, \sigma)$ - the function responsible for the mechanisms of entry of Rn into the chamber, in the general case, can be nonlinear; $K(t-\sigma)$ - memory 
function, determines the degree of influence of the function $F(A, \sigma)$ on the accumulation process; $A_{0}$ - at the initial moment of time $t$.

Note that there can be two limiting cases: when $K(t-\sigma)=\delta(t-\sigma), \delta($.$) - Dirac function,$ in this case there is no memory effect by analogy with Markov processes, and when $K(t-\sigma)=t-H(t-\sigma), H($.$) - the Heaviside function, in this case, characterizes the$ presence of ideal memory. In general, memory function $K(t-\sigma)$ in equation (1) can be selected based on experimental data or properties of the medium.

Due to the fact that the rate of radon intake decreases with time, the faster, the smaller the AER of the chamber with the atmosphere, then the dynamic system (1) will be considered dissipative. Here, by dissipation, we mean that the system gradually "forgets" the mechanisms of the entry of radon into the chamber, i.e. RVA over time goes into a steady state (saturation). For this, in the dissipative system (1) the memory function $K(t-\sigma)$ can be selected in the form:

$$
K(t-\sigma)=\frac{1}{\Gamma(\alpha)(t-\sigma)^{1-\alpha}}, \quad 0<\alpha \leq 1,
$$

where $\alpha$ - determines the intensity of dissipation, $\Gamma($.$) - Euler's gamma function, t \in[0, T]$ - time, $T$ - model time.

Substituting relation (2) into equation (1), we obtain:

$$
\frac{d A}{d t}=I_{0 t}^{\alpha} F(A, \sigma), \quad A(0)=A_{0},
$$

where $I_{0 t}^{\alpha} F(A, \sigma)=\frac{1}{\Gamma(\alpha)} \int_{0}^{t} \frac{F(A, \sigma)}{(t-\sigma)^{1-\alpha}} d \sigma$ - fractional integral of order $\alpha$. If we invert equation (3) taking into account the compositional property of the fractional integral [8], we obtain the following equation:

$$
\partial_{0 t}^{\alpha} A(t)=F(A, \sigma), \quad A(0)=A_{0} .
$$

where

$$
\partial_{0 t}^{\alpha} A(t)=\frac{1}{\Gamma(1-\alpha)} \int_{0}^{t} \frac{\cdot-1-,}{(t-\sigma)^{\alpha}} d \sigma
$$

fractional Gerasimov-Caputo derivative of order $0<\alpha<1,{ }_{i_{1},}, \ldots, d t$.

Note that if in (4) we choose the function $F(A, t)=S_{D}-\lambda_{0} A(t)$, where $S_{D}$ diffusion mechanism of penetration RVA, $\lambda_{0}$ - air exchange rate AER, we will get the results of work [10]. Let's choose a more general function $F(A, t)=-a(t) A^{2}(t)-\lambda_{0} A(t)+S_{D}(t)$, where $0<a(t)<1, S_{D}(t)$ - continuous on the segment $[0, T]$ functions. It should be noted that the first term in the function $F(A, t)$ responsible for slowing down the accumulation of radon in the chamber. Then we arrive at the following Cauchy problem:

$$
\partial_{0 t}^{\alpha} A(t)=-a(t) A^{2}(t)-\lambda_{0} A(t)+S_{D}(t), \quad A(0)=A_{0} .
$$

The Cauchy problem (6) was solved numerically using the modified Newton method with local first-order convergence [11]. 


\section{Simulation results}

The data on the accumulation of radon in the chambers at the PRTR point (Kamchatka), the YSSR point on Sakhalin Island [9] and also GLL (Kamchatka) [3] were used as the initial data for modeling. At these points, continuous registration of radon was carried out in chambers at a depth of 1 to $3 \mathrm{~m}$ [3]. The chambers are a 10 liter bucket. A radon sensor is placed in this chamber through a special pipe. The minimum air exchange between the chamber and the atmosphere is also carried out through the pipe.

The simulation results were compared with the data on the accumulation of radon in the chamber at the GLL point, with an estimate of the determination coefficient between the data and the model curves.

Greatest coefficient of determination $R^{2}=0.89$ was obtained for the following values of the parameters of problem (6), the coefficients were chosen as follows: $a(t)=c(t)=S_{D}(t)=t\left(1-A_{\max } \lambda_{0}\right) / T, \alpha=b=\lambda_{0}=0.25, T=1314, A_{0}=0.484$. The simulation results are shown in the figure.

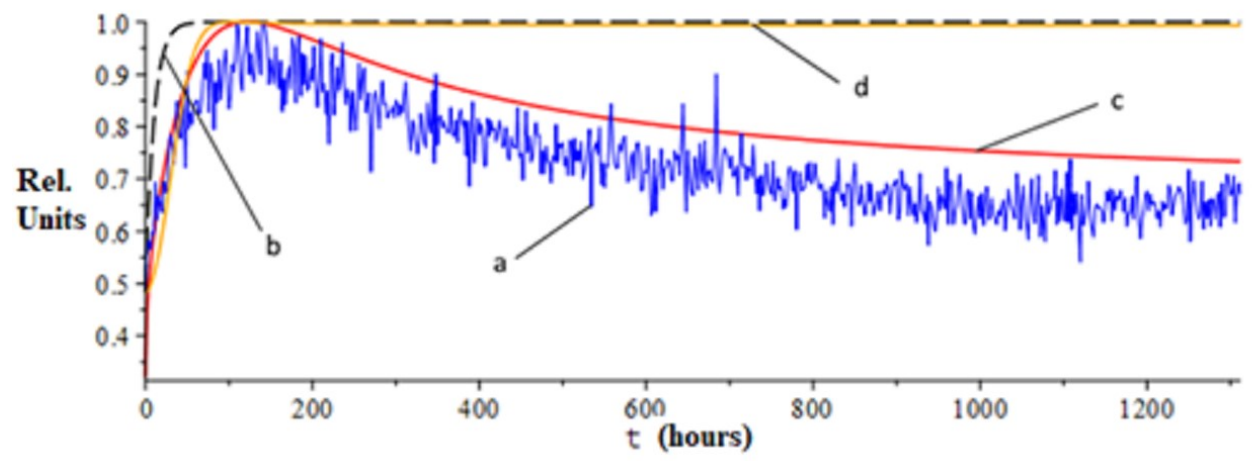

Figure 1. Curves of radon accumulation in the chamber of the GLL point and model curves. a - radon accumulation curve in the chamber at the GLL point; $b$ - calculated curve obtained in [2]; $\mathrm{c}$ - calculated curve obtained by model (6) with $R^{2}=0.89 ; \mathrm{d}$ - calculated curve obtained by model (6) at $\alpha=1, a=c=\lambda_{0}$.

In the figure, all the calculated curves were normalized to the maximum. As can be seen from the figure, the nonlinearity in model (6) makes it possible to obtain new distribution curves of RVA, much closer to the experimental ones, in comparison with the models $[3,10]$.

\section{Conclusion}

The article proposes a new mathematical model of RVA accumulation taking into account heredity. It is shown that the proposed model, taking into account the nonlinearity, can, due to additional parameters: the intensity of dissipation of the accumulation mechanism, give results that are closer to real data, in comparison with the model from [3] and the hereditary model in [10]. 


\section{Acknowledgement}

The work was carried out within the framework of the research project of the Vitus Bering Kamchatka State University Natural disasters of Kamchatka --- earthquakes and volcanic eruptions (monitoring forecast, study, psychological support of the population) No. AAAAA19-119072290002-9 and subject research IKIR FEB RAS "Physical processes in the system of near space and geospheres under solar and lithospheric influences" No AAAAA21-121011290003-0, the work was funded by RFBR, project number 20-05-00493.

\section{References}

1. P.P. Firstov, E.O. Makarov, Seismic instruments. 51, 4 (2015)

2. R.D. Cicerone, J.E. Ebel, J. Beitton, Tectonophysics. 476, 3-4 (2009)

3. P.P. Firstov, E.O. Makarov, Subsoil radon dynamics in Kamchatka and strong earthquakes (KamSU named after Vitus Bering, Petropavlovsk-Kamchatsky, 2018)

4. A.V. Vasilyev, M.V. Zhukovsky, Journal of environmental radioactivity. 124 (2013)

5. R.I. Parovik B.M. Shevtsov, Math. Models and Computer Simulations. 2, 2 (2010)

6. R.I. Parovik, Life Science Journal. 11, 9 (2014).

7. V.V. Uchaikin, Fractional derivatives for physicists and engineers. Volume I (Springer, Berlin, 2013)

8. A.A. Kilbas, H.M. Srivastava, J.J. Trujillo, Theory and Applications of Fractional Differential Equations (Elsevier, Amsterdam, 2006)

9. E. Makarov, P. Firstov, D. Kostylev, E. Rylov, I. Dudchenko, Vestnik KRAUNC. Fiz.-Mat. Nauki., 25, 5 (2018)

10. D.A. Tverdyi, R.I. Parovik, E.O. Makarov, P. P. Firstov, E3S Web Conf. 196 (2020)

11. R.I. Parovik, D.A. Tverdyi, Mathematical and Computational Applications. 26, 3 (2021) 